Editorial

\title{
Acknowledgment to Reviewers of Membranes in 2020
}

\author{
Membranes Editorial Office
}

MDPI AG, St. Alban-Anlage 66, 4052 Basel, Switzerland

Peer review is the driving force of journal development, and reviewers are gatekeepers who ensure that Membranes maintains its standards for the high quality of its published papers. Thanks to the cooperation of our reviewers, in 2020, the median time to first decision was 12 days and the median time to publication was 31 days. The editors would like to express their sincere gratitude to the following reviewers for their precious time and dedication, regardless of whether the papers were finally published:

Abatangelo, Giovanni

Abbrent, Sabina

Abdala, Ahmed

Abdel-Fattah, Essam

Abdellatif, Abdelmohsan

Abd-Elnaiem, Alaa

Abejón, Ricardo

Abraham, Nader

Abuin, Graciela

Achiou, Brahim

Adomavičiūtè, Erika

Afsari, Morteza

Agarkov, Dmitrii

Ahmad, Mohd Zamidi

Airenne, Tomi T.

Akhtar, Sultan

Alaimo, Alessandro

Alayande, Abayomi Babatunde

Albo, Jonathan

Alcoutlabi, Mataz

Alexovič, Michal

Ali, Aamer

Ali, Gomaa

Alique, David

ALjlil, Saad A.

Alkasrawi, Malek

Allegretti, Chiara

Amissah, Felix

Amr, Abd El-Galil E.

An, Chunjiang

Andersson, Jakob

Ang, Micah Belle Marie Yap

Annesini, Maria Cristina

Anokhina, Tatiana S.

Ansari, Mohamed Adam
Antunes, Joana C.

Apollo, Nicholas

Appetecchi, Giovanni Battista

Arnusch, Christopher J.

Artini, Cristina

Arunkumar, Abhiram

Arvanitis, Antonios

Ashrafi, Amirmansoor

Asif, Muhammad Bilal

Attia, Mohamed F.

Attri, Pankaj

Awayda, Mouhamed

Ayyavoo, Jayalakshmi

Azhar, Muhammad Rizwan

Bacchin, Patrice

Bae, Byung-Chan

Bagnato, Giuseppe

Bagolini, Alvise

Bai, Hongwei

Baig, Umair

Bakandritsos, Aristides

Baldanzi, Gianluca

Balme, Sébastien

Ban, Yujie

Banat, Fawzi

Bandini, Serena

Bao, Yongming

Barelli, Hélène

Barloková, Danka

Baschetti, Marco Giacinti

Baschir, Laurentiu

Bassin, Joao Paulo

Bassyouni, Mohamed

Bayer, Ilker

Bazhenov, Stepan D. 
Bazzarelli, Fabio

Becker, Maik

Beckingham, Bryan

Bekiari, Vlasoula

Bellanger, Gilbert

Belosludtsev, Konstantin

Belousov, Valery V.

Bento, Fatima

Bermeshev, Maxim

Bernroider, Gustav

Bernuy-Lopez, Carlos

Bi, Lei

Biçer, Yusuf

Bildyukevich, Alexandr

Bin Darwish, Nawaf

Blandin, Gaetan

Bobacka, Johan

Bocharov, Eduard V.

Bogacki, Jan

Bohinc, Klemen

Bolotov, Leonid

Borisov, Ilya

Bostyn, Stéphane

Bôta, Attila

Bounaceur, Roda

Bramini, Mattia

Braun, Ralf

Breite, Daniel

Brown, Rhoderick E.

Bruno, Nicholas C.

Bruno, Rosaria

Bryjak, Marek

Buchoux, Sebastien

Bulgariu, Laura

Busana, Mattia

Butylskii, Dmitrii

Cabrales, Luis

Calabrò, Francesco

Calafel, Itxaso

Calvo, José Ignacio

Cama, Jehangir

Cametti, Cesare

Cannon, Richard

Cao, Bing

$\mathrm{Cao}, \mathrm{Bo}$

Cao, Liuyue

Carmela, Conidi

Carraro, Mauro

Carreon, Moises

Casado-Coterillo, Clara

Cassano, Alfredo
Castro-Dominguez, Bernardo

Cavallaro, Giuseppe

Cavayas, Yiorgos Alexandros

Cecconet, Daniele

Cevik, Emre

Chae, Sung Ho

Chakraborty, Sudip

Chamberland, Julien

Chang, Hsuan

Charfi, Amine

Chen, George

Chen, Yi-Chun

Chen, Yih-Sharng

Chen, Zhitong

Cheong, Heesun

Chew, Nick Guan Pin

Chew, Thiam Leng

Chiao, Yu-Hsuan

Cho, Kangwoo

Cho, Younghyun

Choi, Jane Ru

Choi, Yong Jun

Chu, Xiangping

Chuah, Chong Yang

Chung, Neal Tai-Shung

Chung, Sheng-Heng

Chwojnowski, Andrzej

Ciura, Krzesimir

Clematis, Davide

Clodt, Juliana Isabel

Coduri, Mauro

Colli, Alejandro Nicolás

Cortina, Jose Luis

Costa, Ana

Costa, Carlos Miguel

Cowan, Matthew Greig

Crespo, João

Cretin, Marc

Crick, Colin R.

Cruz-Alcalde, Alberto

Csanádi, József

Cuccovia, Iolanda Midea

Cui, Zhaoliang

Czernel, Grzegorz

Czogalla, Aleksander

Darling, Seth B.

Daumer, Marie Line

David, Oana

De Angelis, Maria Grazia

De Filpo, Giovanni

De Meijer, Mari 
De Oliveira Lobo, Anderson

De Vos, Wiebe M.

De Vries, Adrianus J.

Deimede, Valadoula

Dema, Roman

Deng, Huining

Deng, Shihai

Derbal, Kerroum

Derbeli, Mohamed

Deshpande, Gauravi

Di Bella, Gaetano

Di Profio, Gianluca

Diddens, Diddo

Diederichsen, Kyle

Ding, Bin

Ding, Yifu

Dinu, Maria Valentina

Ditl, Pavel

Dmitrenko, Mariia E.

Do, Jing-Shan

Dobrzynski, Piotr

Dodero, Andrea

Dołowy, Krzysztof

Dong, Dengpan

Dong, Liangliang

Dong, Weibing

Dong, Xianping

Dong, Xuecheng

Dong, Yingchao

Donzelli, Elisabetta

Dos Reis, Rodrigo Azevedo

Doutch, James

Dudek, Gabriela

Dulova, Niina

Dumee, Ludovic

Duskey, Jason Thomas

Dutta, Ravi Chandra

Ebrahimi, Mehrdad

Edomwonyi-Otu, Lawrence C

Eigenberger, Gerhart

Einkauf, Jeffrey D.

Ekielski, Adam

Ekwemalor, Kingsley

Eliseeva, Tatiana

El-Naas, Muftah H.

Elsaid, Khaled

ElSherbiny, Ibrahim M.A.

Emeline, Perrier-Groult

Esche, Erik

Escorihuela, Jorge

Esmaili, Mansoore
Espíndola, Jonathan Cawettiere

Esposito, Elisa

Estay, Humberto

Evangelopoulos, Michael

Ewers, Helge

Fan, Hongwei

Fan, Senqing

Farshchi Yazdi, Seyed Amir Fouad

Fatyeyeva, Kateryna

Faustino, Vera

Feng, Hongbo

Feng, Qicheng

Feng, Shilun

Fievet, Patrick

Fijałkowski, Karol

Filippov, Anatoly

Fimbres Weihs, Gustavo

Finke, Jan Henrik

Fiorenza, Roberto

Firpo, Giuseppe

Fischer, Kristina

Flanagan, Ted

Fologea, Daniel

Fontalvo, Javier

Fontananova, Enrica

Foti, Claudia

Fraguas-Sánchez, Ana Isabel

Francesco, Galiano

Franco, Camilo Andres

Fryczkowska, Beata

$\mathrm{Fu}, \mathrm{Ziao}$

Funato, Yosuke

Fuoco, Alessio

Gaber, Timo

Galateanu, Bianca

Gan, Shiyu

Gao, Chunmei

Gao, Yanzi

Garab, Győző

Garcia, Andreina

Garcia-Bernabé, Abel

Gareev, Kamil

Gascooke, Jason

Gazdzicki, Pawel

Geffroy, Pierre-Marie

Geisler, Ramsia

Genduso, Giuseppe

Georgieva, Radostina

Georgopanos, Prokopios

Ghalei, Behnam

Ghica, Mihaela Violeta 
Ghiorghita, Claudiu Augustin

Ghoshal, Shraboni

Giannotti, Marina Inés

Gierszewska, Magdalena

Giuffre, Ottavia

Gizdavic-Nikolaidis, Marija

Glasmacher, Birgit

Glowinska, Ewa

Goddard, Alan

Goh, Kunli

Goh, Pei Sean

Gomez-Coma, Lucia

Gonzales, Ralph Rolly

González, Jose

González-López, Tomás José

Gościańska, Joanna

Gozálvez-Zafrilla, José Marcial

Gradov, Oleg M.

Grahovac, Jovana

Green, Matthew

Green, Michael

Grushevenko, Evgeniia

Gryta, Marek

Grzegorzek, Martyna

Gu, Qilin

$\mathrm{Gu}$, Zhi-Gang

Guan, Kecheng

Gubler, Lorenz

Gueorguiev, Gueorgui K.

Guibal, Eric

Guillén, Elena

Gulari, Erdogan

Güler, Enver

Gulicovski, Jelena

Gumieniczek, Anna

Gurkan, Burcu

Guskov, Albert

Gutierrez-Aguilar, Manuel

Gzara, Lassaad

Habuda-Stanic, Mirna

Haddad, Maryam

Hagar, Mohamed

Hamzah, Azrul Azlan

Han, Runlin

Han, Xiuguo

Hanasoge, Srinivas

Handge, Ulrich A.

Harayama, Takeshi

Harja, Maria

Hart, Kyle

Hartkamp, Remco
Hartman, Mariusz

Harun, Mohd Hamzah

Hasan, Abdel Fattah R.

Hashim, Khalid

Hashimoto, Masanori

Hassan, Mohammad K.

Hassouna, Mohamed Salah El-Din

$\mathrm{He}$, Wei

He, Zeming

Helmi, Arash

Hernández, Agustín

Hernandez-Aldave, Sandra

Heumann, Rolf

Hidalgo, Asuncion Maria

Hirota, Yuichiro

Hlaibi, M.

Ho, Jia Shin

Hofmann, Andreas

Hollmann, Axel

Homaeigohar, Shahin

Hong, Jin Gi

Hong, Liang

Hong, Tao

Horbett, Thomas A

Hosui, Atsushi

Hou, Jingwei

Hou, Shangguo

Hou, $\mathrm{Yi}_{\mathrm{i}}$

Hsien, Tzu Yang

Hsu, Jyh-Ping

$\mathrm{Hu}$, Anming

$\mathrm{Hu}$, Chengzhi

$\mathrm{Hu}$, Jian

$\mathrm{Hu}$, Ning

Huang, Guosheng

Huang, Manhong

Huertas, Rosa

Hyun, Dong Choon

Ifelebuegu, Augustine

Ihsanullah, Ihsanullah

Iijima, Kazutoshi

Ilavsky, Jan

Iliev, Boyan

Imbrogno, Alessandra

Insepov, Zinetula (Zeke)

Iorio, Ronald M.

Islam, Syed Z.

Ismail, Manal

Itta, Arun

Ivancev-Tumbas, Ivana

Ivanets, Andrei 
Iwata, Tomoyuki

Jacomin, Anne-Claire

Janagam, Dileep R.

Jansen, Johannes

Jastrzab, Renata

Jen, Tien Chien

Jeon, Ju-Won

Jeong, Euikyoung

Jeong, Kwanho

Jeong, Sung-in

Jho, Eun Hea

Ji, Guozhao

Ji, Yan-Li

Jia, Da

Jia, Hongge

Jiang, Chenxiao

Jiang, Junke

Jiang, Qiu-Xing

Jiang, Tao

Jin, Bo

Jin, Congrui

Jiu, Yaming

Jobin, Marie-Lise

Johnson, Colin P.

Joo, Jonghoon

Jørgensen, Mads Koustrup

Joshi, Rakesh

Jovanović, Zoran

Jue, Melinda L.

Jung, Eun Sang

Kagramanov, Georgiy

Kahar, Prihardi

Kalantari, Katayoon

Kalantar-zadeh, Kourosh

Kaleekkal, Noel Jacob

Kallem, Parashuram

Kallunki, Tuula

Kammakakam, Irshad

Kanematsu, Hideyuki

Kang, Byung-jae

Kang, Moon-sung

Kang, Sang Wook

Kang, Yang Jun

Kanno, Takahiro

Kappert, Emiel

Karabagias, Ioannis K.

Karavasili, Christina

Karimi, Mohsen

Karkhanehchi, Hamed

Karuppasamy, K.

Karvan, Oguz
Kashif, Muhammad

Kaškonienė, Vilma

Kemperman, Antoine

Kertèsz, Szabolcs

Khan, Mohammad Ehtisham

Khan, Mohammed Nazeer

Kharkar, Prathamesh

Kikionis, Stefanos

Kim, Ae Rhan

Kim, Albert

Kim, Hanki

Kim, Keugtae

Kirichenko, Ksenia

Kiss, Eva

Kitamura, Akira

Kitchen, Philip

Klaysom, Chalida

Koeppe, Roger E.

Kokosa, John M.

Kolya, Haradhan

Kong, Xian

Konieczkowska, Jolanta

Kononova, Svetlana V.

Koprowski, Piotr

Kore, Rajkumar

Korolkov, Ilya V.

Kostoglou, Margaritis

Koter, Stanislaw

Kothandaraman, Jotheeswari

Koukounaras, Athanasios

Koutahzadeh, Negin

Kovacs, Renato

Kovács, Zoltán

Kowalczyk, Tomasz

Kowalik-Klimczak, Anna

Kowalska, Ewa

Kozmai, Anton Eduardovich

Kraslawski, Andrzej

Krieg, Henning

Krumova, Sashka

Kshatri, Aravind

Kujawa, Joanna

Kujawski, Wojciech

Kulda, Vlastimil

Kulesha, Olga

Kumar Thakur, Vijay

Kurihara, Masaru

Kurlyandskaya, Galina

Kuroda, Daisuke

Kurtz, Ira

Kusuma, Victor A. 
Kuyukina, Maria S.

Kvasha, Andriy

Kyriakopoulos, Grigorios L.

La Rosa, Angela D.

Lade, Harshad S.

Laguna-Bercero, Miguel Ángel

Lai, Gwo-Sung

Laín, Santiago

Lamas-Samanamud, Gisella

Lameloise, Marie-Laure

Lammertink, R.G.H.

Lanč, Marek

Laoui, Tahar

Lasseuguette, Elsa

László, Zsuzsanna

Latorrata, Saverio

Lau, Woei Jye

Lavric, Vasile

Le, Zaiyuan

Le-Clech, Pierre

Lee, Albert

Lee, Eui-Jong

Lee, Hyun Su

Lee, Jae-Suk

Lee, Jaewoo

Lee, Kew-Ho

Lee, Kuo Hao

Lee, Pyung Soo

Lee, Sungyun

Lehocký, Marián

Lei, Weiwei

Lenik, Joanna

Li, Chengyong

Li, Hui

$\mathrm{Li}$, Jianxin

Li, Junsheng

Li, Lu Hua

Li, Shiben

Li, Wanbin

Li, Yuanchao

Li, Zhipeng

Li, Zidong

Liang, Shuang

Lillepärg, Jelena

Lin, Haiqing

Lin, Hongjun

Lin, Shuo

Lin, Weifeng

Lin, Weiyun

Lin, Xubo

Linares, Jose
Ling, Zheng

Lipińska, Andrea

Lipnizki, Frank

Lipscomb, Glenn

Liu, Baijun

Liu, Bo

Liu, Dongxia

Liu, Gongping

Liu, Guicai

Liu, Hua-Min

Liu, Huolong

Liu, Junyi

Liu, Minghua

Liu, Pengqing

Liu, Shaomin

Liu, Shyh-Jiun

Liu, Xianhu

Liu, Yang

Liu, Yong

Lobo, Anderson

Long, Rui

López, Julio

Loulergue, Patrick

Low, Nicholas

Lu, Peng

$\mathrm{Lu}$, Wei

Lundin, Sean-Thomas B.

Lunguleasa, Aurel

Luo, Fabao

Luo, Tao

Ma, Hongjuan

Ma, Jiangya

Ma, Tianji

Ma, Xiaoli

Macedo, Antónia

Macedonio, Francesca

Magalhães, Leandro

Magnacca, Giuliana

Magnico, Pierre

Makisha, Nikolay

Małolepszy, Artur

Mancini, Marco

Marchetti, Laura

Maréchal, Manuel

Mareev, Semyon

Marquês, Joaquim Manuel Trigo

Martí-Calatayud, Manuel César

Martínez Rodrigo, Javier

Masoudi Soltani, Salman

Matějka, Milan

Mateus, Marília 
Matharu, Rupy Kaur

Matko, Vojko

Matsumoto, Michiaki

Matsuura, Takeshi

Mayyahi, Ahmed Al

Mažeikienè, Aušra

Mazinani, Saeed

Mazor, Ohad

Mazurkiewicz-Pawlicka, Marta

Mazzei, Rosalinda

Mazziotti Di Celso, Giuseppe

Melin, Frederic

Melnikov, Stanislav

Meng, Fanbin

Meng, Shujuan

Meng, Xiangchao

Mihai, Mara

Mikhelson, Konstantin

Miller, Daniel N.

Minakshi, Manickam

Minamiki, Tsukuru

Miranda, Adelaide

Miranda, João Mário

Mitkowski, Piotr

Mizsey, Peter

Moghaddam, Saeed

Molina, Serena

Molnar, Oleksandr

Mondal, Sayan

Monjezi, Alireza Abbassi

Monnot, Mathias

Monteiro, Bernardo

Montoro, Carmen

Morozan, Adina

Morozova, Sofia

Moteki, Takahiko

Mousa, Hamouda

Moutloali, Richard

Mozo, Juan Danie

Mukherjee, Maitreyee

Mukhopadhyay, Kausik

Mullaliu, Angelo

Müller, Rüdiger

Murmura, Federica

Murmura, Maria Anna

Murphy, Eoin G.

Muschiol, Jan

Muthumareeswaran, M.R.

Nabil, Bouazizi

Nagamine, Kuniaki

Nagarkar, Amit A.
Nagul, Edward A.

Nagy, Endre

Nagy, Miklós

Nair, Vaishakh

Nakahara, Hiromichi

Nakielski, Pawel

Nam, Sang Yong

Nasir, Rizwan

Nastase, Florin

Natale, Paolo

Naumowicz, Monika

Nechifor, Gheorghe

Nędzarek, Arkadiusz

Nemestóthy, Nándor

Nesterkina, Mariia

Neuman, Manuela

Nevárez-Moorillón, Guadalupe Virginia

Nicoletta, Fiore Pasquale

Nikolaeva, Daria

Nishihara, Masamichi

Nogueira, António

Nomura, Mikihiro

Ntougias, Spyridon

Nuez, Ignacio

Nunna, Bharath Babu

Nyamutswa, Lavern T.

Ochoa, Nelio Ariel

Oh, Hee Jeung

Oh, Hyun-Suk

Oh, Jonghyun

Ohya, Yoshikazu

Okamoto, Yoshiyuki

Oliva, Rosario

Oren, Yoram

Orfi, Jamel

Oron, Gideon

Orooji, Yasin

Ortiz Martínez, Víctor Manuel

Ortiz-Medina, Josue

Oumi, Yasunori

Ovalle-Encinia, Oscar

Pacheco Tanaka, David Alfredo

Pacheco, Federico

Padil, Vinod V. T.

Padilla-Benavides, Teresita

Paganin, Valdecir Antonio

Paglieri, Stephen

Pal, Animesh

Palacio, Laura

Palumbo, Oriele

Panepinto, Deborah 
Panglisch, Stefan

Pani, Marcella

Paolone, Annalisa

Papadakis, Raffaello

Park, Jin Yong

Park, Jin-Soo

Park, Taehyun

Parnian, Mohammad Javad

Parrino, Francesco

Pasquini, Luca

Patel, Rajkumar

Patrauchan, Marianna A.

Patsios, Sotiris

Pawlowski, Sylwin

Pedersen-Bjergaard, Stig

Peer, Petra

Peng, Hao

Peng, Xueyuan

Peng, Yong

Peng, Yuan

Pergher, Sibele

Pernyeszi, Tímea

Peters, Thijs

Petriev, Iliya

Pham, Chuyen Van

Piotr, Szczepanski

Pirzada, Tahira

Pisk, Jana

Pismenskaya, Natalia

Plisko, Tatiana V.

Poerio, Teresa

Polańczyk, Andrzej

Ponomarev, Igor I.

Popovic, Svetlana

Post, Jan

Pouliot, Yves

Pradanos, Pedro

Prasad, Vikram

Prieto, Manuel

Prisic, Sladjana

Pruncu, Catalin I.

Pulyalina, Alexandra

Puszkiel, Julián

Qanud, Khaled

Qasim, Muhammad

Qiang, Yuhao

Qin, Kairong

Qin, Yu

Raceanu, Mircea

Radu, Aleksandar

Rafiq, Sikander
Rahimpour, Mohammad Reza

Rajca, Mariola

Ramadoss, Ananthakumar

Rambabu, Gutru

Rao, Sanjeev

Rätzke, Klaus

Reinosa, Julián Jiménez

Reis, Cristiano

Reis, Miria Hespanhol Miranda

Restivo, João

Rider, Andrew N.

Rincón, Marina E.

Robert, Philippe A.

Rodrigues Reis, Cristiano E.

Rodriguez De San Miguel, Eduardo

Ronen, Avner

Rosenberger, Sandra

Roubík, Hynek

Roy, Sagar

Ruan, Xuehua

Ruiz-García, Alejandro

Ruthstein, Sharon

Rybak, Aleksandra

Saakes, Michel

Sachdeva, Ir. Sumit

Sadmani, A.H.M. Anwar

Saeki, Daisuke

Sahoo, Sanjubala

Samal, Sangram

Samhaber, Wolfgang M.

Samsudin, Asep Muhamad

Sánchez Navarro, Amparo

Sankarasubramanian, Shrihari

Santhoshkumar, Puttur

Santoro, Sergio

Santos Juanes-Jorda, Lucas

Santos, Rafael M.

San-Valero, Pau

Sanyal, Oishi

Sapalidis, Andreas

Savić, Jelena

Sawamura, Kentaro-ichi

Scarazzato, Tatiana

Scheepers, Fabian

Schiraldi, Chiara

Schirhagl, Romana

Schneck, Emanuel

Schuster, Bernhard

Schwantes, Rebecca

Seçkin, Turgay

Seddighi, Sadegh 
Semino, Rocio

Sengupta, Debarun

Seong, Jong Geun

Sequeira, César A. C.

Sessa, Lucia

Setničková, Katerina

Sevcsik, Eva

Severino, Patrícia

Sezgin, Erdinc

Sgreccia, Emanuela

Shafieian, Abdellah

Shaik, Mohammed Rafi

Shao, Jiahui

Shao, Lu

Sharma, Priyanka R.

Shaulsky, Evyatar

Shellaiah, Muthaiah

Shen, Jiangnan

Shevate, Rahul

Shi, Zheng

Shimoga, Ganesh

Shin, Sehyun

Shtepliuk, Ivan

$\mathrm{Si}$, Yang

Siekierka, Anna

Sikhwivhilu, Keneiloe

Sillence, Dan J.

Silva, Mónica

Sim, Lee Nuang

Simari, Cataldo

Sinha Ray, Saikat

Sinha Ray, Sumit

Sioutopoulos, Dimitrios

Sivakumar, Mani

Skinner, Jack

Skorik, Yury A.

Slouka, Zdenek

Snow, Samuel

Soboloff, Jonathan

Song, Xiaoxiao

Song, Young Eun

Sousa, Sílvia A.

Soveral, Graça

Stamatialis, Dimitrios

Staszak, Maciej

Stojmenović, Marija

Stolarczyk, Agnieszka

Strandbakke, Ragnar

$\mathrm{Su}$, Baowei

$\mathrm{Su}$, Jincai

$\mathrm{Su}, \mathrm{Xiao}$
Subach, Fedor V.

Subramanian, Palaniappan

Sun, Ramon

Sung, Gun Yong

Sysel, Petr

Szaferski, Waldemar

Sze, Lai Li

Szekely, Gyorgy

Szeleszczuk, Łukasz

Szwast, Maciej

Szymański, Kacper

Takamuku, Shogo

Tańczyk, Marek

Tang, Kewen

Tang, Yuan-Hui

Tanioka, Akihiko

Tarditi, Ana Maria

Téllez, Carlos

Teo, Jeremy

Teodosiu, Carmen

Teplyakov, Vladimir

Teter, Ken

Thakur, Vijay Kumar

Thekkiniath, Jose

Thibault, Jules

Tian, Miao

Tijing, Leonard

Tiraferri, Alberto

Tiwale, Nikhil

Tocci, Elena

Toh, William

Tomašič, Tihomir

Tong, Xin

Torem, Maurício Leonardo

Tosheva, Lubomira

Toth, Andras Jozsef

Tres, Marcus Vinicius

Tringe, Joseph

Tripathy, Sunil Kumar

Trocino, Stefano

Tsagarakis, Konstantinos

Tsehaye, Misgina Tilahun

Tseng, Hui-hsin

Tsuge, Hideaki

$\mathrm{Tu}$, Qingsong

Tufa, Ramato Ashu

Tundidor-Camba, Alain

Tung, Kuo-Lun

Tuti, Simonetta

Uchihashi, Takayuki

Ursino, Claudia 
Urtenov, Makhamet

Václavíková, Natália

Vaiano, Vincenzo

Valachová, Katarína

Vallabhajosyula, Saraschandra

Van Der Bruggen, Bart

Van Goethem, Cédric

Vanangamudi, Anbharasi

Vandezande, Pieter

Vardhan, Harsh

Vasos, Paul

Versaci, Mario

Vinothkannan, Mohanraj

Visser, Tymen

Vlaicu, Ioana Dorina

Volkov, Alexey

Volkov, Vladimir

Vranes, Milan

Vrouwenvelder, Johannes

Wagh, Priyesh

Wagh, Priyesh A.

Waldron, Kevin

Wang, Chih-Min

Wang, David

Wang, Guorong

Wang, Hongsheng

Wang, Jian

Wang, Jie

Wang, Jun (Jiangsu University of Science

and Technology)

Wang, Jun (Nanjing University)

Wang, Kean

Wang, Lei

Wang, Liguo

Wang, Naixin

Wang, Qi

Wang, Tonghua

Wang, Xuefen

Wang, Yaoming

Wang, Zhaoxu

Wang, Zhi

Wang, Zhiwei

Wang, Zhongpeng

Wawrzkiewicz, Monika

Wei, Chun-hai

Wei, Gang

Wei, Junchao

Wei, Yanying

Wei, Zhishun

Weinman, Steven T.

Weng, Guo-Ming
Wenten, I. Gede

Wieland, D. C. Florian

Witt, Katarzyna

Włodarczyk, Paweł P.

Wong, Danny K. Y.

$\mathrm{Wu}$, Bing

Wu, Gwo-Mei

$\mathrm{Wu}$, Lili

$\mathrm{Wu}$, Rome-Ming

$\mathrm{Wu}$, Yinghao

Wycisk, Ryszard

Wylie, Benjamin J.

Xiang, Yuan

Xiao, Kang

Xin, Dongyue

Xiong, Xin

$\mathrm{Xu}$, Tao

$\mathrm{Xu}, \mathrm{Xin}$

$\mathrm{Xu}$, Zhiwei

Xue, Shuangmei

Yamaki, Tetsuya

Yan, Haiyang

Yan, Kai

Yang, $\mathrm{Hu}$

Yang, Ruey-Jen

Yang, Wei

Yin, Ben Hang

Yin, Yan

Yntema, Doekle

Yoo, Dong Jin

Yourey, William

Yu, Dawei

$\mathrm{Yu}$, Haifeng

$\mathrm{Yu}$, Jaecheul

$\mathrm{Yu}$, Tzyy Leon

Yuan, Zhizhang

Yuan, Zhongyun

Yun, Yanbin

Yurekli, Yilmaz

Zagklis, Dimitris

Zarca, Gabriel

Zarybnicka, Lucie

Zarzycki, Arkadiusz

Zeng, Lin

Zhang, Hongbo

Zhang, Huacheng

Zhang, Junliang

Zhang, Liqun

Zhang, Wenxiang

Zhang, Yajie 
Zhang, Yanlin

Zhang, Yizhou

Zhang, Zhongqiang

Zhao, Shuaifei

Zhao, Yulong

Zheng, Libing

Zholobko, Oksana

Zhou, Jian

Zhou, Tao

Zhou, Xu

Zhou, Zhengzhong
Zhu, Guanghui

Zhu, Jie

Zhu, Lingxiang

Zhu, Meihua

Zhu, Shan

Žigon, Jure

Zolotukhin, Denis B.

Zoltan-Istvan, Szabo

Zouboulis, Anastasios

Zuo, Jian 